Meta

Journal des tradlucteurs

Translators' Journal

\title{
Traductions, théories et traducteurs en U.R.S.S.
}

\section{Vladimir Grebenschikov}

Volume 12, numéro 1, mars 1967

URI : https://id.erudit.org/iderudit/002964ar

DOI : https://doi.org/10.7202/002964ar

Aller au sommaire du numéro

Éditeur(s)

Les Presses de l'Université de Montréal

ISSN

0026-0452 (imprimé)

1492-1421 (numérique)

Découvrir la revue

Citer cet article

Grebenschikov, V. (1967). Traductions, théories et traducteurs en U.R.S.S. Meta, 12(1), 3-8. https://doi.org/10.7202/002964ar d'utilisation que vous pouvez consulter en ligne.

https://apropos.erudit.org/fr/usagers/politique-dutilisation/ 


\section{TRADUCTIONS THÉORIES ET TRADUCTEURS EN U.R.S.S.}

\section{PAYS DE LA TRADUCTION}

L'U.R.S.S. est actuellement le pays de la traduction par excellence: on y compte plus de deux cents langues très différentes et plus de vingt littératures nationales. D'après les statistiques de l'O.N.U., l'U.R.S.S. est aussi le pays où se publie le plus grand nombre de traductions. D'ailleurs la traduction a toujours joué un rôle prépondérant dans la formation et le développement des civilisations nationales qui font partie de l'U.R.S.S. d'aujourd'hui. Dès les $I^{e}$ et $x^{e}$ siècles, les Russes furent constamment en contact avec des langues appartenant à des familles fort diverses: grec moyen et latin, langues germaniques, turques, finno-ougriennes, caucasiennes, iraniennes, mongoles, sémitiques, baltiques, slaves et autres. Des documents multiples attestent la présence d'une tradition longue et vigoureuse en traduction littéraire: traductions du grec byzantin au $\mathrm{XI}^{\mathrm{e}}$, du latin et de l'allemand aux $\mathrm{xv}^{\mathrm{e}}$ et $\mathrm{XVI}^{\mathrm{e}}$ siècles, et traduction des vers syllabiques polonais au XVII ${ }^{\mathrm{e}}$ siècle. Mais c'est surtout au XVIII ${ }^{\mathrm{e}}$ siècle que la traduction prend son véritable essor, grâce aux réformes d'« occidentalisation» de l'empereur Pierre $\mathbf{I}^{\text {er }}$. C'est par des essais de traduction de textes littéraires français, puis allemands et, plus tard, anglais et espagnols, que les premiers écrivains professionnels russes ont d'abord commencé à exercer leur métier.

Après la révolution de 1917 , la traduction dans les langues nationales, autres que le russe, commence à se développer. La Russie compte actuellement plusieurs milliers de traducteurs professionnels, dont le tiers environ travaillent à traduire des langues occidentales vers le russe. On fait des efforts particuliers pour intensifier les traductions des langues asiatiques et africaines ${ }^{1}$.

Ce bref panorama nous fait entrevoir l'importance et l'intérêt de l'expérience de la traduction en U.R.S.S. Pourtant il est étonnant qu'on ne se soit éveillé qu'assez tard, soit depuis le milieu du siècle, à la théorie de la traduction. De plus, des considérations purement doctrinales - qui faisaient rejeter, par exemple, les principes de la linguistique structurale et quelques autres découvertes de la linguistique occi-

1. V1. Rossels, «Za eti gody », Masterstvo perevoda, Moskva, Sov. pisatel, 1959, p. 227.

Vol. $12-\mathrm{N}^{\circ} \mathrm{I}$ 
dentale - ont assez longtemps freiné les recherches en vue d'une théorie fondamentale.

Depuis 1954 toutefois, on peut observer une évolution de la situation: un certain nombre de travaux intéressants ont cherché à analyser la riche expérience acquise, et des recherches théoriques et pratiques ont porté sur la traduction littéraire et la traduction automatique.

\section{PREMIERS PAS \\ VERS \\ UNE THÉORIE DE LA TRADUCTION}

En 1953 paraît à Moscou l'Introduction à une théorie de la traduction, de A.V. Feodorov, première tentative visant à jeter les bases linguistiques d'une théorie générale de la traduction ${ }^{2}$. L'ouvrage a suscité une réaction assez vive dans les diverses revues et chez les traducteurs professionnels du pays ${ }^{3}$. Deux ans plus tard paraît sous la signature d'un certain nombre de traducteurs soviétiques éminents ${ }^{4}$ un recueil d'articles qui s'attaquent violemment aux principes linguistiques mis en avant par Feodorov.

Ainsi se dessinent les deux courants fondamentaux de la conception de la traduction en U.R.S.S., et l'on peut dire que les tenants de deux théories se livrent une lutte acharnée.

La majorité des traducteurs qui ont pris position semble se rallier à la théorie « esthétique et intuitive ». Le véritable traducteur littéraire est un artiste, insistentils. L'inspiration ne peut être contrôlée ni mise en cage - la cage linguistique ne fait pas exception à la règle ${ }^{5}$. Les meilleures traductions littéraires sont l'œuvre d'écrivains, de poètes ou d'artistes-traducteurs comme Pouchkine, Joukovsky, Tourgueniev, Gneditch, Bounine, Pasternak et autres, qui ignoraient jusqu'à l'existence d'une science linguistique $^{6}$. Les conceptions linguistiques de la traduction mènent au «bukvalisme » (littéralisme), gâchis digne d'un artisan et non d'un artiste ${ }^{\top}$. Le traducteur doit rechercher non pas l'exactitude linguistique de son travail, mais la conformité aux réalités sociales de l'œuvre qu'il traduit, car « le traducteur soviétique est le plus avancé des propagandistes de l'amitié entre les peuples ${ }^{8}$. On n'hésite pas à invoquer l'opinion de traducteurs occidentaux qui s'opposent à l'élaboration d'une théorie linguistique de la traduction ${ }^{9}$.

En 1958 paraît une seconde édition, revue et augmentée, du livre de A.V. Feodorov, portant en sous-titre « Problèmes linguistiques ». L'auteur insiste avec vi-

2. Cf. A.V. Feodorov, Vvedenie v teoriu perevoda, Moskva, 1953, et Vvedenie v teoriu perevoda (Lingvistitcheskie problemy), Moskva, 1958, $375 \mathrm{p}$.

3. F. Feldmann, Voprosy yazykoznania, $\mathrm{n}^{\circ} 2$, 1954, p. 117-127; L. Borovoy, «Poetika perevoda $\gg$, Druzhba narodov, no 4, 1954, p. 249-253.

4. Cf. Voprosy khoudojestvennogo perevoda, Moskva, Sov. pisatel, 1955, qui contient sept essais signés par P. Antokolsky, P. Toper, A. Leites, I. Kachkine, Vl: Rossels, O. Kundzitch, L. Sobolev.

5. P. Antokolsky, M. Aouezov, M. Ryssky, ibid., p. 9-10.

6. P. Toper, « Tradicii realisma $»$, ibid., p. 45-96.

7. A. Kundzitch, «Perevodcheskaya mys1 i perevodcheskoye nedomyslie», ibid., p. 213-220; Masterstvo perevoda, Moskva, Sov. pisatel, 1959, p. 7-44 et, spécialement, p. 25.

8. S. Lipkine, « Perevod i sovremennost», Masterstvo perevoda, Moskva, Sov. pisatel, 1963 p. 14-15.

9. Edmond Cary, la Traduction dans le monde moderne, citê par P. Antokolsky, Masterstvo perevoda, Moskva, Sov. pisatel, 1959, p. 456-459. 
gueur sur la primauté de l'aspect linguistique de la traduction qui fait qu'on ne saurait élaborer une théorie générale de la traduction sans recourir aux recherches linguistiques ${ }^{10}$. Feodorov met à contribution l'immense documentation que constituent les traductions littéraires en U.R.S.S. Mais il ne dépasse pas les cadres de la linguistique traditionnelle et ne tient même pas compte des innovations apportées par Ferdinand de Saussure et Charles Bally. Cependant, traducteurs et théoriciens $\mathrm{du}$ courant linguistique manifestent de plus en plus de vigueur: un nombre toujours croissant de savants et de traducteurs publient des travaux d'analyse linguistique des méthodes de traduction.

Les deux courants continuent toujours à s'affronter. Chaque camp publie de nombreux travaux de recherche, fondés sur une riche expérience et abondamment illustrés d'exemples explicites, vivants et souvent très convaincants.

\section{PROGRÈ DES RECHERCHES \\ ET \\ DES TRAVAUX DE TRADUCTION}

Il y a eu apparemment certaines tentatives de conciliation afin de modérer le zèle des opposants. C'est dans cet esprit qu'on a organisé en 1956-1957 une série de conférences et de séminaires dans les grands centres culturels du pays ${ }^{11}$. Ces initiatives se sont révélées très fructueuses, si bien que des conférences de ce genre se tiennent maintenant en assez grand nombre avec une périodicité variable. Les travaux de ces conférences sont publiés de temps à autre sous forme d'actes. Par exemple, les actes annuels de l'Art de la traduction ${ }^{12}$ qui contiennent des articles très intéressants sur un grand nombre de problèmes propres à la traduction. Le courant antilinguistique semble y prédominer. On y trouve fréquemment cependant des recherches et des analyses de caractère purement linguistique. Mentionnons encore, danis la même veine, les Cahiers du traducteur ${ }^{13}$ et les actes de la première conférence universitaire de la traduction, tenue à l'Université de Leningrad, du $1^{\text {er }}$ au 6 juin $1951^{14}$. Ces actes comprennent des travaux et des analyses sur des problèmes linguistiques, la stylistique comparée, la lexicographie, l'histoire et la théorie de la traduction. Ici toutefois, c'est le courant linguistique qui domine avec, en particulier, L. Barkhoudarov, A.V. Feodorov et Ia. Retsker. L'article d'introduction, signé par B.A. Larine, représente une tendance nouvelle, celle du compromis: « La théorie de la traduction est impensable sans une liaison organique des méthodes linguistiques et littéraires... La traduction est une variété fonctionnelle du bilinguisme dont elle a toutes les caractéristiques, en plus de comporter, au niveau supérieur, des exigences esthétiques qui tiennent de la théorie littéraire. ${ }^{15}$

10. A.V. Feodorov, Vvedenie v teoriu perevoda, Moskva, 1958, p. 4.

11. En mars 1954, à Kiev (Ukraine); en juin 1956, à Ufa (Bachkirie); en mars 1957, à Tallin (Estonie); au printemps 1957, à Leningrad et à Moscou. Cf. V1. Rossels, « Za eti gody 》, Masterstvo perevoda, Moskva, Sov. pisatel, 1959, p. 214-218.

12. Masterstvo perevoda. Sbornik statey, Moskva, Sov. pisatel. Ces volumes d'actes ont paru en $1955,1959,1963,1964$ et 1965 . On y trouve les noms des auteurs les plus actifs: $P$. Antokolsky, I. Kachkine, V1. Rossels, P. Toper, O. Kundzitch, E. Etkind, Ag. Gatov, V. Levik, L. Ozerov et autres.

13. Tetradi perevodchika, Moskva. Les deux premiers cahiers avaient été publiés en 1960 et en 1961 par le Premier Institut pédagogique de langues étrangères à Moscou. Trois autres cahiers, publiés en 1963, 1964 et 1965, le furent par l'Institut des relations étrangères.

14. Cf. Teoria i kritika perevoda, Leningrad, Leninragskij Universitet, 1962, $168 \mathrm{p}$.

15. B.A. Larine, « Nachi zadatchi $\gg, i b i d .$, p. 4.

$\underset{\text { Mars } 1967}{\text { Vol. } 12} \mathbf{N}^{\circ}, 1$ t 


\section{STATUT DU TRADUCTEUR}

ET

DE LA TRADUCTION

Ã leur conférence annuelle de 1956, les traducteurs de Moscou avaient élaboré des «propositions pour l'amélioration du travail des traducteurs » ${ }^{16}$. Apparemment un certain nombre de ces propositions ont été appliquées, mais on ne sait pas encore si les traducteurs russes ont obtenu pour leur profession un statut officiel. Certaines de ces propositions visaient nettement cet objectif:

- Au cours de conférences régulières, favoriser l'étude par les traducteurs euxmêmes des problèmes généraux de la traduction et de la profession.

- Création de séminaires de traduction en divers endroits pour la formation des jeunes traducteurs par les traducteurs expérimentés.

- Création d'une section de traduction littéraire à l'Institut littéraire Maxime Gorki à Moscou pour assurer le recrutement de cadres compétents.

- Mention obligatoire du nom de l'auteur d'une traduction (articles, livres). Démarches en ce sens auprès des organismes de publication et de publicité, ainsi qu'auprès de la Direction générale de publication (Glavizdat).

- Demande au Glavizdat en vue de mesures de protection des droits d'auteur des traducteurs et pour l'interdiction des abus existants:

a) changement arbitraire du tirage.

b) changement de l'ordre de paiement des honoraires.

c) modifications des honoraires établis.

d) réédition d'œuvres traduites sans l'accord préalable du traducteur.

e) modification des manuscrits sans l'approbation de l'auteur-traducteur.

- Demande pour que des traducteurs professionnels fassent partie du Conseil d'administration de toutes les entreprises qui publient des œuvres traduites.

- Interdiction de l'engagement de plusieurs traducteurs pour traduire une même œuvre, si ces traducteurs ne font pas équipe; interdiction de la pratique courante de désigner arbitrairement des coauteurs au traducteur engagé.

- Imposition à toutes les maisons d'édition d'un « code unique de publication des œuvres littéraires traduites ». Ce code, rédigé sous l'autorité du Glavizdat, devrait comprendre les points suivants:

a) autoriser les éditeurs à conclure des contrats directement avec les traducteurs pour une période maximale de trois ans.

b) interdire la publication de traductions avant qu'elles soient vérifiées et comparées aux originaux; réglementer les normes de travail des rédacteurs des œuvres traduites et limiter le temps accordé à la vérification des textes traduits. c) exiger que les contrats signés par les traducteurs se conforment aux normes suivantes: pour un traducteur littéraire, production maximale de quatre feuilles de traduction en prose et cinq cents vers de poésie par mois; en règle générale, un seul traducteur pour une cuvre en prose ou en vers (jusqu'à concurrence de 2000 vers).

\section{NOUVEAUX CADRES PROFESSIONNELS}

Le recrutement et la formation de cadres professionnels sont parmi les ques-

16. Vl. Rossels, op. cit., p. 223-227; A. Argo, « Fakty i vyvody », Masterstvo perevoda, Moskva, Sov. pisatel, 1959, p. 295-301. 
tions les plus importantes présentement débattues par les traducteurs russes, l'augmentation du volume d'œuvres à traduire ayant créé un besoin pressant de traducteurs.

Avant 1956, on s'était peu occupé de la formation des traducteurs. Les traducteurs novices se formaient d'eux-mêmes ou sous la surveillance d'un traducteur expérimenté. Ils se recrutaient parmi les diplômés soit des facultés de philologie, de littérature ou de linguistique, soit des instituts de langues étrangères ${ }^{17}$.

La division des opinions sur la théorie de la traduction se retrouve aussi à propos de la formation du traducteur. Les partisans de la conception esthétique ou intuitive prônent la formation des jeunes traducteurs au moyen de «séminaires » où les jeunes s'exercent à traduire sous la direction de traducteurs chevronnés. Ivan Kachine et Korney Tcgoukobsky, entre autres, ont ainsi fait école. À Leningrad, la section des traducteurs, au sein de l'Union des écrivains soviétiques, a mis en marche plusieurs séminaires de traduction littéraire de ce genre. Les éditeurs de la ville se sont engagés à n'employer que des jeunes traducteurs recommandés par les directeurs des séminaires en question.

Les «linguistes » de leur côté s'efforcent de créer des écoles de formation de traducteurs, dotées de programmes fonctionnels et systématiques dont l'armature repose sur des principes scientifiques. La première expérience en ce sens semble être la fondation en 1956 d'une section de traduction littéraire auprès de l'Institut littéraire Maxime Gorki à Moscou. Cette section se vit confier la tâche de former de 150 à 200 traducteurs professionnels pour la traduction vers le russe d'œuvres appartenant à trente littératures étrangères. Le programme élaboré s'échelonne sur cinq ans. Il comprend, en plus du «cycle philologique », des cours sur l'économie, la sociologie, l'histoire et la civilisation du pays, selon la spécialisation des candidats. Des séminaires de formation intensive, théorique et pratique, complètent le programme ${ }^{18}$.

L'exemple de l'Institut Maxime Gorki a été bientôt suivi par d'autres universités: Tbilissi, Erevan, Leningrad, Tachkent, Minsk, Tartou, entre autres, ont formé des facultés ou des départements de traduction littéraire ${ }^{19}$.

\section{LINGUISTIQUE STRUCTURALE ET}

\section{TRADUCTION AUTOMATIQUE}

Cette brève revue de la situation en U.R.S.S. ne serait pas complète si l'on ne faisait mention du livre de I.I. Revzine et Iu. Rozentsveig, les Fondements de la traduction traditionnelle et automatique ${ }^{20}$. C'est le dernier en date des travaux consacrés aux recherches théoriques en traduction.

Ce livre donne l'essentiel du cours de théorie de la traduction donné en 19591961 à la Faculté de traduction du Premier Institut pédagogique de langues étrangères à Moscou. Le cours vise essentiellement à nouer organiquement les éléments de la théorie linguistique aux techniques mises au point en traduction automatique.

17. Vl. Rossels, op. cit., p. 228.

18. V1. Rossels, op. cit., p. $230-231$.

19. P. Antokolsky, « Tchornyi khleb masterstva », Masterstvo perevoda, Moskva, Sov. pisatel, 1963, p. 11.

20. Osnovy obstchego i machinnogo perevoda, Moskva, Vyschaya chkola, 1964, 243 p. 
Le pont se fait naturellement par la linguistique structurale. Cette orientation représente un nouveau courant de pensée et de recherche en U.R.S.S. Les auteurs vont jusqu'à reprocher à A.V. Feodorov et $\mathrm{A}$. Reformatsky de ne pas pousser assez loin leur théorie linguistique de la traduction. Ils soutiennent que l'utilisation des techniques de l'information en linguistique et en traduction peut contribuer énormément à l'élaboration d'une théorie scientifique et objective de la traduction.

Les auteurs concluent évidemment que tout programme de formation de traducteurs professionnels devrait inclure des éléments de linguistique structurale, d'analyse automatique de la langue et de traduction automatique.

VLADIMIR GREBENSCHIKOV 